\title{
Successfully Treated Severe Acne Using Selective Electrothermolysis in a Patient with Nephrotic Syndrome
}

\author{
Hwa Jung Ryu, Ji Hyun Park, Sook In Ryu, Bo Young Kim, Seung Hyun Chun \\ Department of Dermatology, Ansan Hospital, Korea University College of Medicine, Ansan, Korea
}

Acne is very common in adolescents and young adults. Although there are various conventional treatment modalities, some patients are prone to side effects and need alternative options. A 22-year-old male patient who were treated with high dose systemic steroid for his nephrotic syndrome, encountered severe acne on his face and neck. Since the patient's medical condition was unable to administer systemic agent, he was treated with the selective electrothermolysis device. The sebaceous gland targeting treatment by selective electrothermolysis with microneedle radiofrequency device, had minimal adverse effect and the skin lesions improved dramatically. The patient was satisfied and did not want further treatment. (Ann Dermatol 31(1) $75 \sim 77,2019$ )

\section{-Keywords-}

Acne vulgaris, Sebaceous gland, Selective electrothermolysis, Radiofrequency therapy

\section{INTRODUCTION}

Acne is one of the most common inflammatory skin dis-

Received June 5, 2017, Revised November 16, 2017, Accepted for publication January 4, 2018

Corresponding author: Hwa Jung Ryu, Department of Dermatology, Korea University Ansan Hospital, 123 Jeokgeum-ro, Danwon-gu, Ansan 15355, Korea. Tel: 82-31-412-5180, Fax: 82-31-412-4208, E-mail: dermhj@naver.com ORCID: https://orcid.org/0000-0003-2136-4682

This is an Open Access article distributed under the terms of the Creative Commons Attribution Non-Commercial License (http://creativecommons. org/licenses/by-nc/4.0) which permits unrestricted non-commercial use, distribution, and reproduction in any medium, provided the original work is properly cited.

Copyright (c) The Korean Dermatological Association and The Korean Society for Investigative Dermatology orders affecting the pilosebaceous unit. Adolescents are predominantly affected, but it can persist into adulthoods. Pathogenesis of acne is multifactorial, and several factors such as follicular hyperkeratinization, increased sebum production, Propionibacterium acnes, and inflammation are well known. Although there are various treatment modalities, it is difficult to cure acne due to its multifactorial pathogenesis. Conventional therapies include topical agents, systemic antibiotics, and systemic retinoid. However microbial resistance, irritation, and systemic adverse effects are possible. Patients who are particularly prone to those side effects need alternative options. Recently, a new therapeutic modality of selective electrothermolysis targeting the sebaceous glands was introduced ${ }^{1}$.

\section{CASE REPORT}

A 22-year-old male presented with skin lesions on his face and neck. On physical examination, numerous comedonal papules and erythematous pustules were seen on his mandible, anterior and both lateral sides of his neck (Fig. 1A). He had been diagnosed as nephrotic syndrome two year ago. In the nephrology clinic he was treated with oral methylprednisolone for five months. His skin lesions began during this systemic steroid therapy, and they did not get better after tapering off of the steroid medication. The patient encounterd psychologic stress because of his skin findings. After referral to our dermatology clinic, a topical benzoyl peroxide/clindamycin gel was applied for 1 month, but the patient complained of no improvement. However, his nephrotic syndrome recurred and he had to begin treatment with deflazacort, a glucocorticoid prodrug. Since the patient's medical condition was unable to taper steroid nor administer minocycline by then, we decided to treat him with an elective electrothermolysis 


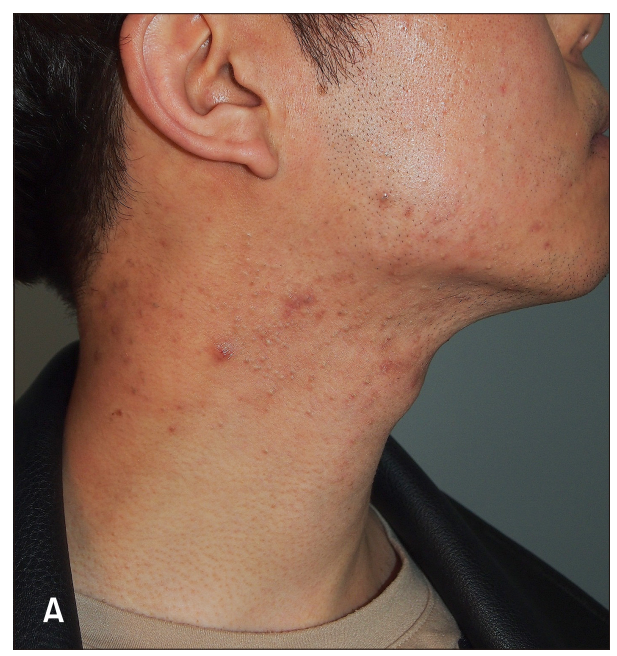

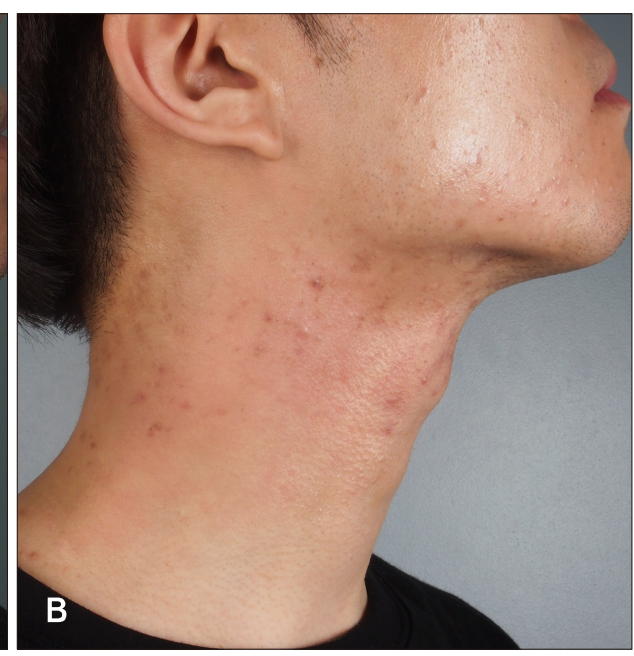

Fig. 1. Severe comedonal acne in 22-year-old male. (A) Before the selective electrothermolysis treatment. (B) Final photo after 3 treatment sessions. device (AGNES micro-insulated needles with RF applicators; Gowoonsesang Dermatology Clinic, Seoul, Korea) to alleviate his skin symptoms. We received the patient's consent form about publishing all photographic materials. A topical anesthetic cream was applied, and comedonal lesions were gently extracted with a needle and acne extractor. A tip of a $1.5 \mathrm{~mm}$ microinsulated needle (I-type) was inserted into the center of the pore, and the high frequency current was applied about two or three times at an energy level of $1 \mathrm{MHz}$ radiofrequency (RF). The day after the procedure, the remaining contents of the lesions were extracted again. The treatment was performed at one month intervals. After three treatment sessions, the skin lesions improved dramatically without any side effects (Fig 1B). The patient was satisfied and did not want further treatment.

\section{DISCUSSION}

Acne can be challenging in some patients, whose acne does not respond to conventional therapies. Severe acne can have psychological effects and leave scarring. Various factors such as hormonal change, stress and some medications are known to aggravate conditions and lead to severe acne. Topical or systemic steroid is a well-known causative agent for steroid acne ${ }^{2}$.

Patients with severe acne often have aggravating factors and simultaneously have a potential risk of side effects from systemic antibiotics or retinoids. However they ask clinicians for any available treatment options. New modalities targeting acne have been adopted such as photodynamic therapies ${ }^{3}$, nonablative radio frequency devices, and selective electrothermolysis ${ }^{4}$.

The conventional treatment targeting sebaceous glands has the disadvantage of producing indirect damage to the epidermis. However, the recently introduced selective electrothermolysis with a minimally invasive microneedle RF device overcomes such problems by creating radiofrequency thermal zones with minimal epidermal injury. Preliminary evidence was suggested by Kobayashi and Tamada1 for facial seborrhea. An animal study of the rabbit ear acne model demonstrated effective destruction of sebaceous glands, and also reduction in the levels of inflammatory mediators such as tumor necrosis factor- $\alpha$ after treatment with micro-insulated needles with $\mathrm{RF}^{5}$.

In this case, the treatment was minimally invasive, caused tolerable pain and downtime of less than 2 days with immediate erythema. No adverse effects of swelling, or postinflammatory hyperpigmentation were seen. This outcome was achieved by a well-trained clinician in this case. However there is a possibility of a local burn if the micro-needles are not inserted to a sufficient depth. We report a case of successfully treated severe acne which had persisted after systemic steroid treatment. The method of selective electrothermolysis can be an alternative treatment for young women of child bearing age, patients with renal and liver dysfunction and those who experienced adverse effects of conventional therapies.

\section{CONFLICTS OF INTEREST}

The authors have nothing to disclose.

\section{ORCID}

Hwa Jung Ryu, https://orcid.org/0000-0003-2136-4682

Ji Hyun Park, https://orcid.org/0000-0003-4856-1774

Sook In Ryu, https://orcid.org/0000-0002-9880-4837

Bo Young Kim, https://orcid.org/0000-0002-2650-0391

Seung Hyun Chun, https://orcid.org/0000-0001-7399-5561 


\section{REFERENCES}

1. Kobayashi T, Tamada S. Selective electrothermolysis of the sebaceous glands: treatment of facial seborrhea. Dermatol Surg 2007;33:169-177.

2. Fung MA, Berger TG. A prospective study of acute-onset steroid acne associated with administration of intravenous corticosteroids. Dermatology 2000;200:43-44.

3. Choi JW, Kim TI, Jeong KH, Shin MK. A case of successfully treated recalcitrant EGFR inhibitor-induced acneiform eruption following non-ablative fractional laser. Dermatol Ther 2016;29:252-254.

4. Song BH, Lee DH, Kim BC, Ku SH, Park EJ, Kwon IH, et al. Photodynamic therapy using chlorophyll-a in the treatment of acne vulgaris: a randomized, single-blind, split-face study. J Am Acad Dermatol 2014;71:764-771.

5. Lee JW, Kim BJ, Kim MN, Ahn GY, Aso H. Selective sebaceous gland electrothermolysis as a treatment for acne: a prospective pilot study. Int J Dermatol 2012;51:339-344. 